\title{
TERAPIA DE LINGUAGEM NA AFASIA
}

\author{
MAURO SPINELLI* \\ JAIRZA LAPETINA ** \\ MARIA LUIZA ROBADO ** \\ PEDRO SIMOES ROSADO *.* \\ JOSE GERALDO CAMARGO LIMA ****
}

A capacidade de comunicação com seus semelhantes é fundamental para o bem-estar social, econômico e psicológico do ser humano e os distúrbios desta capacidade impedem a sua participação integral na səciedade. A mais importante forma de comunicação do ser humano se dá através da linguagem escrita e falada que se realizam graças à capacidade de simbolização do cérebro humano. Lesōes cerebrais que provocam falhas na simbolizaçäo, conduzem ao aparecimento do quadro clínico denominado afasia ou disfasia. A causa mais frequente de afasia no paciente adulto, faixa etária a que se refere o presente trabalho, é a patologia vascular cerebral, vindo, em segundo lugar, os tratmatismos crânio-encefálicos.

A preocupação em reintegrar os afásicos à sociedade data de longo tempo e, já em 1890, Bateman (citado por Sarno \& col. ${ }^{10}$ ) sugeria que um meio de restaurar a linguagem seria a "reeducação". Em 1901, Charles Mills (citado por (ruchaga ${ }^{3}$ ) publicou um artigo intitulado "O tratamento dos afásicos pelo treinamento". Muitos anos se passaram, mas até a presente data, ainda prosseguem as divergências de opinião sobre os métodos e os resultados da reeducação da linguagem na afasia. Lenneberg ${ }^{8}$ considera que o paciente adulto não reaprende a linguagem mediante processos de aprendizagem, apoiando a posição de Godfrey \& Douglas 5 que não vêem diferença entre resultados espontâneos e resultados atribuídos a processos de reeducação da linguagem. Luria ${ }^{9}$ e Vignolo ${ }^{14}$ apresentam casuística na qual a boa evolução não pode ser atribuída à recuperação espontânea. Cruchaga ${ }^{3}$ e Schuell $\&$ col. ${ }^{12}$ consideram que a terapia de linguagem na afasia é mais uma arte do que uma ciência e que os seus resultados são de difícil quantificaçäo. Eles sugerem que o debate sobre a efetividade da terapia provavelmente não terá fim, pois a questão envolve um número muito grande de variáveis, o que impede a comparação dos resultados

Trabalho do Departamento de Neurologia e Neurocirurgia da Escola Paulista de Medicina, da Disciplina de Foniatria da Pontificia Universidade Católica de São Paulo e do Centro de Distúrbios da Audição e Linguagem de Săo Paulo, apresentado no VII Congresso Brasileiro de Neurologia (Salvador, Bahia, 18-22 julho, 1976):* Professor de Foniatria da Pontificia Universidade Católica de săo Paulo; * Fonoaudióloga do Centro de Distúrbios da Audiçăo e Linguagem de São Paulo: *** Médico Estagíário da Cínica Neurológica da Escola Paulista de Medicina; *** Professor Adjunto da Clínica Neurológica da Escola Paulista de Medicina. 
obtidu: por diferentes autores. Esta posição é bastante pessimista se forem analisados os esforços e avanços da patologia da comunicação, no sentido de tornar objetivos os seus procedimentos e avaliaçōes.

A indicação ou não de terapia de linguagem para afásicos tem sido feita com base em resultados de testes, como o Functional Comunication Profile (FCP), preconizado por Sarno \& col. ${ }^{10}$ e outros métodos que permitem traçar $\checkmark$ perfil iuncional do paciente e, assim, determinar o tipo e grau de perturbações que ele apresenta. Autores como Butfield \& Zangwill 2 e Sands \& col. ${ }^{11}$ consideram que o prognóstico é mau nos casos. severos. Godfrey \& Douglas ${ }^{5}$ e Cruchaga ${ }^{3}$ são alguns que não consideram vantajosa a terapia em casus com muito comprometimento da recepção. Todavia, há estudos, como 0 de Vignolo ${ }^{14}$, demonstrando haver boa evolução em casos severos receptivos.

Quanto à idade, cs afásicos idosús têm sido considerados de mau prognósîico (Ciodirey \& Douglas "). Eisenson 4 observa que os melhores resultados são ubtidos em jovens, mas não exclui a indicação de terapia nos casos de pessoas idosas. $O$ inicio da terapia, para Beyn ${ }^{1}$, Leche ${ }^{7}$, Vignolo ${ }^{14}$ e Seffer ${ }^{13}$, deve ser o mais precoce possivel, desde que as condições clinicas do paciente o permitan. Assim há a vantagem de se ir de encontro a uma possivel recuperação esontânea, o que apressa a evolução, além de evitar indesejáveis fenômenos de adaptação que ocorrem em todo o afásico após algum tempo.

$\mathrm{O}$ métocio a ser utilizado depende do tipo e grau de afasia, alén de outros fatores de menor importância. Não existem "fórmulas" ou métodos-padrões.

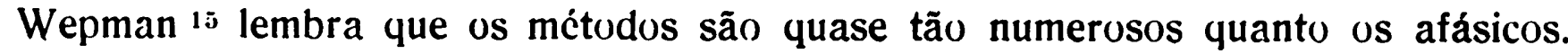
$O$ plano de terapia deve ser adaptado a cada paciente e a grande maioria usa alguma forma de estimulação auditiva, como afirmam Sarno \& col. 10. As metas a atingir também influem na escolha do método. Se o terapeuta se preocupa exclusivamente com a fala, terá maiores possibilidades de obter sucesso mediante técnicas estritamente programadas, como as descritas por Holland \& Harris '. Se o terapeuta encara, como um problema psicolinguístico, psiconeurológico e psico-social e visa a aperfeiçoar a linguagem e auxiliar 0 afásico na reintegração social, seu método deverá incluir menos técnicas dirigidas aos aspectos articulatórios e mais procedimentos livres, sob a forma de diálogos.

As bases neurofisiológicas da terapia de linguagem são discutidas por Cruchaga ${ }^{3}$ que considera haver estimulação de funções deficitárias, tal como se atua frente a um membro parético, e ainda a utilização de reflexos condicionados e de automatismos latentes. Luria" enfatiza o papel do terapeuta no sentidn Ue colaborar para a reorganização de sistemas funcionais, pelos quais o resultauo final da atuação de sistemas orgânicos pode ser obtido de forma aceitável, embora de formas diferentes das utilizadas habitualmente. As bases psicológicas da terapia são apresentaads por Wepman ${ }^{13}$ e Eisenson * que atribuem à motivação e à desinibição, papéis fundamentais para a recuperação do afásico.

Para Beyn ${ }^{1}$, Cruchaga ${ }^{3}$ e Seffer ${ }^{13}$ a duração da terapia deve ser longa, no mínimo de um ano, em sessões diárias ou em dias alternados. As sessões não devem ser demoradas pará não cansar os pacientes. Beyn ${ }^{1}$ ainda sugere que, após o término do plano de terapia, um programa de manutenção, com 
sessões a intervalos de três a quatro meses, seria útil para impedir que o paciente venha a perder as funções linguisticas readquiridas. A avaliação dos resultados da terapia é feita, em geral, utilizando-se o método ou teste empregado na avaliação inicial e comparando os escores obtidos pelo paciente, antes e depois da terapia.

\section{MATERIAL E METODOS}

Indicamos a terapia de linguagem para todos os casos de afasia, desde que năn sejam devidos a lesáo evolutiva. Iniciamos o tratamento o mais precocemente possivel. Os fundamentos teóricos de nossa estratégia são basicamente os de Eisenson 4. Luria \& e Wepman 15.

Para elaborar o plano terapéutico, estudamos a comunicação oral e escrita; a atividade social e profissional; a personalidade do paciente; a sua familia; a audiçâ. tonal, discriminação de sons da fala. retencăo e evocaçăo auditiva. controle postural e motor dos órgáos da fala.

Para o aperfeiçoamento da compreensăo da fala, partimos do nivel em que se encontra o paciente, usando fala apenas lentificada, pausada nos casos leves, e usando repetiçăo de simples vocábulos nos casos severos. Técnicas para o aperfeiçoamenton da percepção e da memórla såo utilizadas. Para o aperfelçoamento da emisåáo oral, utilizamos procedimentos dírigidos à reconstruçán de esquemas motores e outros destinados a facilitaçăo da evocaçăo verbal e da organizaçăo da sintaxe em graus diferentes. de acordo com a necessidade do caso.

Utilizamos sessões de 45 minutos, duas vezes por semana, em média, com atendimentos individuais a em grupos. Os grupos săo organizados após conhecermos bem as caracteristicas dos pacientes, de forma a reunir os individuos que tenham boas possibilidades de se adaptarem bem.

Estudamos retrospectivamente 15 casos de afasia, atendidos em terapla de linguagem e selecionados segundo o critério único de estarem bem documentados quanto ans aspectos que nos interessavam especialmente, e que eram: idade, sexo, tipo de patologia, típo de afasia, intervalo entre o inicio do quadro e início da terapia, duraça da terapia, resultado obtido.

As patologias responsáveis pela afasia foram: acidente vascular cerebral em 11 casos e traumatismos encefálicos por acidentes ou como complicaçăo de cirurgía em 4 casos. Oito pacientes eram do sexo masculino e sete do sexo feminino. Quanto a idade, tinham menos de 10 anos, 1 caso; de 10 a 20 anos, 2 casos; de 20 a 30 anos. 3 casos; de 30 a 40 anos, 3 casos; de 40 a 50 anos, 2 casos; mais de 50 anos. 4 casos.

Quanto ao tipo de afasia, todos os pacientes apresentavam algum grau de comprometimento da recepça oral. Cinco tinham alteraçóes predominantemente expressivas. os outros dez apresentavam déficits receptivos e expressivos em graus equiparávela. configurando quadros homogeneos.

Quanto ao intervalo entre o aparecimento da afasia e o início da terapia, seis casos foram atendidos durante o periodo em que há recuperaçáo espontanea relevante, isto é, dentro dos três meses seguintes à eclosåo do problema. A duraça da terapia variou de 1 a 30 meses.

\section{RESULTADOS}

Para avaliarmos os resultados foram utilizados critérios referentes a comunicaçá e critérios relacionados à modificaçós emocionais e à integraçáo soclal. Năo nos deteremos nos aspectos emocionais e sociais, citando apenas que, nesse aspecto, 10 pacientes obtiveram bons resultados e 5 permaneceram inalterados. Quanto à comuni- 
caçăo oral, avaliamos os déficits no inf́cio e no final do período terapêutico, segundo níveis de compreensão e de expressăo, com determinacão da média obtida nesses dois itens.

Caracterizamos os seguintes níveis de compreensão: 0 - compreensăo ausente: 1 - compreensão de itens muito simples, como responder ao nome e a ordens simples desde que se repita duas ou mais vezes a frase; 2 - compreensão de ordens simples na primeira emissăo, desde que relacionadas à situação atual, com auxilio: 3 - compreensão de ordens simples sem auxilio e de conversaçăo sobre tópicos de muita relevância para o paciente; 4 - compreensão de or Jens semi-complexas e de explicaçós a respeito de material e técnicas usadas na terapia. Compreensão de conversação referente a tópicos năo presentes na situaçăo, exigindo fala lentificada e redundante: 5 - compreensão de conversaçáo informal e de ordens complexas com falhas ao serem incluidos tópicos abstratos, fala rápida ou com mudanças bruscas de conteúdo: 6 comprensăo boa, sem falhas a não ser em testagens aprofundadas.

Para emissão, caracterizamos os níveis: 0 - emissão com significado auscnte. Po le haver ausência total de emissóes; 1 - emissão de palavras isoladas, inteligibilidade muito pouco eficiente, com ou sem jargåo; 2 - emissão de frases curtas ou de fala muito telegráfica, com uso de vocabulário reduzido, de sintaxe alterada, ou dispraxia, muito prejudiciais para a inteligibilidade; 3 - emissão razoavelmente eficiente, mas exigindo conhecimentos do interlocutor para interpretar o significaio, ou repetiçס̃es da emissåo e uso de pistas auxiliares, por parte do paciente. Uso de parafasias, dificuldades de evocaçăo prejudicando a fluência, fala telegráfica, dispraxia; 4 - uso de frases longas e periodos, com dificuldades com o vocabulário e erros de sintaxe que prejudicam pouco a inteligibili-jade. Fala telegráfica esporádica, rodeios (circunlóquius) para compensar as dificuldades de evocação, poucas dificuldades práxicas; 5 - fala inteligivel, com dificuldadies para exprimir idéias complexas, fazer relatos completos. Falhas de evocaçăo e sintáticas esporádicas. Dificuldades com vocabulários abstratos; 6 - fala boa, com falhas aparentes. somente em testagem aprofundada, pouco nitidas na conversaçắ comum.

A avaliação é feita através ce coleta contínua de dados, durante o atendimento. pelo terapeuta e periodicamente pelo médico responsável.

Para a classificação dos casos quanto à gravidade da afasia e quanto ao grau de progresso obtido, usamos o seguinte critério: severos, os casos com médias dos niveis alcançados em recepção e em emissăo, ating,indo de 0 a 2,5; moderados, com médias de 3,0 até 4,0; leves, os casos com média acima de 4,0. Consideramos como homogêneos os casos com diferenças iguais ou menores do que 1,5 entre os niveis receptivo e expressivo. Clessificamos os resultados em ótimos, bons e maus, segundo os progressos observados na avaliação final. Estes progressos foram quantificados através das diferenças entre a média fínal e a média obtida na avaliaçăo inicial da comunicaçăo. Consideramos como ótimos os resultados em que os progressos atingiram 3 pontos ou mais; como bons os resultados com progressos de 1,5 a 2.5 pontos e como maus bs resultados com progressos menores do que $\mathbf{1 , 5}$.

\section{COMENTARIOS}

Baseados nesse critério podemos dizer que, no nosso material de 15 afásicos, 10 apresentavam grau severo, 4 grau moderado e um, grau leve. Dez pacientes apresentavam afasia homogênea e, 5 afasia predominantemeente expressiva.

Embora não se possa fazer uma análise estatística, podemos fazer alguns comentários: dos 15 pacientes afásicos, 11 apresentaram evolução satisfatória ( 6 com bom resultado e 5 com ótimo resultado). Seis desses 11 pacientes tinham iniciado a terapia mais de três meses após a instalação do quadro afásico, o que sugere a eficácia da terapia em si, pois a recuperação espontânea atua pre- 
dominantemente nos três primeiros meses. Desses 11 pacientes que tiveram evolução favorável, 6 tinham afasia do grau severo; desses, três apresentavam o tipo homogêneo, sugerindo que o grau da afasia e o seu tipo, não deve ser critério para eleição dos casos para terapia.

A análise de nosso material, sugere que o prognóstico em terapia da afasia não pode ser rigidamente ligado a fatores isolados, como idade, tipo de patologia e o intervalo entre a instalação do quadro e o início da terapia. A terapia da linguagem no paciente afásico é efetiva, o prognóstico não pode ser feito a priori. Somente a evolução do caso, com sua retestagem criteriosa, poderá dar dados realmente úteis para análise da evolução.

\section{RESUMO}

A terapia da linguagem na afasia foi estudada em 15 pacientes, 8 do sexo masculino e 7 do sexo feminino; a idade variou de menos de 10 anos a mais de 50 anos; em 11 casos a afasia era sequela de acidente vascular cerebral e, em 4, de traunatismo crânio-encefálico. Quanto ao tipo de afasia, 5 pacientes apresentavam déficits predominantemente de expressão e 10 apresentavam déficits receptivos e expressivos em graus equiparáveis. Em 6 casos a terapia foi iniciada dentro dos 3 primeiros meses de instalação do quadro; nos restantes foi iniciada mais tardiamente. Dos 15 pacientes, 11 apresentaram evolução satisfatória, a qual não depndeu do grau de afasia, do tipo de afasia, da idade do paciente, do tipo de patologia e do intervalo entre a instalação do quadro afásico e 0 início da terapia. A terapia da linguagem no paciente afásico é efetiva, o prognóstico não pode ser feito a priori. Somente a evolução do caso com suạ retestagem criteriosa poderá dar dados realmente úteis para se falar em evolução.

\section{SUMMARY}

\section{Speech therapy in aphasia}

The speech therapy in fifteen aphasic patients was studied. The group consisted of 8 male and 7 female patients and their ages ranged from less than ten to more than fifty years. In eleven cases the aphasia was due to cerebrovascular disease and in the remaining four cases to traumatic injury to the brain. Predominantly expressive aphasia accounted for five patients while in the others a comparable degree of receptive and expressive deficits existed. Six patients had their therapy programme started within the first three months after the acute phase and the rest of the group started it after more time had elapsed. Eleven patients showed a satisfactory revovery regardless the inicial deficit severity, the patients age, the pathology of the lesion or the period of time which elapsed from the beginning of the disease to the start of the therapy. The speech therapy in aphasic patients is effective although a firm prognosis cannot be established at the beginning of the programme. Useful information concerning prognosis only can be taken from the follow-up and after thoroughly retesting the patients. 


\section{REFERENCIAS}

1. BEYN, E. S. - Basic principles of restorative therapy of speech in aphasia. Bibl. Psychiatr. Neurol. $139: 174,1969$.

2. BUTFIELD, E. \& ZANGWILL, O. L. Reeducation in aphasia: a review of 70 cases. J. Neurol., Neurosurg. a. Psychiat. 9:75, 1946.

3. CRUChaGA, J. G. - La rehabilitacion de los pacientes af́́sicos. Arq. NeuroPsiquiat. (Săo Paulo) 29:184, 1971.

4. EISENSON, J. - Aphasia in adults. In TRAVIS, L. E. - Handbook of Speech Pathology and Audiology. Appleton-century-crofts, New York, 1971.

5. GODFREY, C. M. \& DOUGLAS, E. The revovery in aphasia. Canadian Med. Ass. J. $80: 618,1959$.

6. HOLLAND, A. L. \& HARRIS, A. B. - Aphasia rehabilitation using programmed instruction: an intensive case history. In SLOANE, H. N. \& MAC AULAY, C. - Operant Procedures in Remedial Speech and Language Training. Miflin, Boston 1968.

7. LECHE, P. - Speech therapy and the treatment of cerebrovascular drseases. Proc. Roy. Soc. Med. 65:85, 1972.

8. LENNEBERG, E. H. - Biological Foundations of Language. John Wiley, New York, 1967.

9. LURIA, A. R. - Restoration of Function after Brain Injury. MacMillan, New York, 1963:

10. SARNO, M. T.; SILVERMAN, M. G. \& SANDS, E. S. - Speech therapy and language recovery in severe aphasia. J. Speech and Hearing Res. 13:607, 1970.

11. SANDS, E. S.; SARNO, M. T. \& SHANKEILER, D. P. - Long-term assessment of language function in aphasia due to stroke. Arch. Physical Me1. a. Rehabilitation 50:202, 1969.

12. SCHUEL, H.; JENKINS, J. J. \& JIMENEZ-PABON, E. - Aphasia in Adults: Diagnosis, Prognosis and Treatment. Hoeber, New York, 1964.

13. SEFFER, J. W. - A case study demonstrating the value of aphasia therapy. British J. Disorders of Comunication, 8:99, 1973.

14. VIGNOLO, L. A. - Evolution of aphasia and language rehabilitation: a retrospective exploratory study. Cortex 1:344, 1964.

15. WEPMAN, J. M. - A conceptual model for the processes involved in recovery from aphasia. J. Speech a. Hearing Disorders, 18:4, 1953.

Departamento de Neurologia e Neurocirurgia - Escola Pauligta de Medicina Caixa Postal 80.391 - 01000 Sdo Paulo, 8P - Brasil. 